\title{
Economics needs a scientific revolution
}

\author{
Financial engineers have put too much faith in untested axioms and faulty models, says Jean-Philippe \\ Bouchaud. To prevent economic havoc, that needs to change.
}

Compared with physics, it seems fair to say that the quantitative success of the economic sciences has been disappointing. Rockets fly to the Moon; energy is extracted from minute changes of atomic mass. What is the flagship achievement of economics? Only its recurrent inability to predict and avert crises, including the current worldwide credit crunch.

Why is this so? Of course, to paraphrase Isaac Newton, modelling the madness of people is more difficult than modelling the motion of planets. But statistical regularities should emerge in the behaviour of large populations, just as the law of ideal gases emerges from the chaotic motion of individual molecules. To me, the crucial difference between modelling in physics and in economics lies rather in how the fields treat the relative role of concepts, equations and empirical data.

Classical economics is built on very strong assumptions that quickly become axioms: the rationality of economic agents (the premise that every economic agent, be that a person or a company, acts to maximize his profits), the 'invisible hand' (that agents, in the pursuit of their own profit, are led to do what is best for society as a whole) and market efficiency (that market prices faithfully reflect all known information about assets), for example. An economist once told me, to my bewilderment: "These concepts are so strong that they supersede any empirical observation." As economist Robert Nelson argued in his book, Economics as

"Classical economics has no
framework through which to
understand 'wild' markets."

Religion (Pennsylvania State Univ. Press, 2002), the marketplace has been deified.

Physicists, on the other hand, have learned to be suspicious of axioms. If empirical observation is incompatible with a model, the model must be trashed or amended, even if it is conceptually beautiful or mathematically convenient. So many accepted ideas have been proven wrong in the history of physics that physicists have grown to be critical and queasy about their own models.

Unfortunately, such healthy scientific revolutions have not yet taken hold in economics, where ideas have solidified into dogmas. These are perpetuated through the education system: students don't question formulas they can use without thinking. Although numerous physicists have been recruited by financial institutions over the past few decades, they seem to have forgotten the methodology of the natural sciences as they absorbed and regurgitated the existing economic lore.

The supposed omniscience and perfect efficacy of a free market stems from economic work done in the 1950s and 1960s, which with hindsight looks more like propaganda against communism than plausible science. In reality, markets are not efficient, humans tend to be over-focused in the short-term and blind in the long-term, and errors get amplified, ultimately leading to collective irrationality, panic and crashes. Free markets are wild markets.

\section{Picture imperfect}

Reliance on models based on incorrect axioms has clear and large effects. The Black-Scholes model, for example, which was invented in 1973 to price options, is still used extensively. But it assumes that the probability of extreme price changes is negligible, when in reality, stock prices are much jerkier than this. Twenty years ago, unwarranted use of the model spiralled into the worldwide October 1987 crash; the Dow Jones index dropped 23\% in a single day, dwarfing recent market hiccups. Ironically, it was the very use of a crash-free model that helped to trigger a crash.

This time, the problem lies, in part, in the development of structured financial products that packaged subprime risk into seemingly respectable high-yield investments. The models used to price them were fundamentally flawed: they underestimated the probability that multiple borrowers would default on their loans simultaneously. These models again neglected the very possibility of a global crisis, even as they contributed to triggering one.

Surprisingly, classical economics has no framework through which to understand

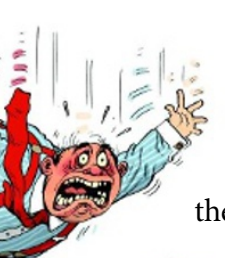
their existence is so obvious to the layman. Physics, on the other $\stackrel{\circ}{\circ}$ hand, has developed several models that explain how small perturbations can lead to wild effects. The theory of complexity shows that although a system may have an optimum state, it is sometimes so hard to identify that the system never settles there. This optimum state is not only elusive, it is also hyper-fragile to small changes in the environment, and therefore often irrelevant to understanding what is going on. There are good reasons to believe that this paradigm should apply to economic systems in general and financial markets in particular. We need to break away from classical economics and develop completely different tools. Some behavioural economists and econo-physicists are attempting to do this now, in a patchy way, but their fringe endeavour is not taken seriously by mainstream economics.

While work is done to enhance models, regulation also needs to improve. Innovations in financial products should be scrutinized, crash-tested against extreme scenarios outside the realm of current models and approved by independent agencies, just as we have done with other potentially lethal industries (chemical, pharmaceutical, aerospace, nuclear energy).

Crucially, the mindset of those working in economics and financial engineering needs to change. Economics curricula need to include more natural science. The prerequisites for more stability in the long run are the development of a more pragmatic and realistic representation of what is going on in financial markets, and to focus on data, which should always supersede perfect equations and aesthetic axioms.

Jean-Philippe Bouchaud is head of research of Capital Fund Management and a physics professor at École Polytechnique in France. e-mail: jean-philippe.bouchaud@cea.fr 